\title{
Role of Biorepositories in Personalized Medicine: Isolation and Processing of Primary Tumor Cells for the initiation Tumor Cultures
}

Zhang H, Ma J, Wang J, Moore W, Liang Z.

Biomedical Engineering from Beijing Institute of Technology, China

*Corresponding Author : Zhang H , Biomedical Engineering from Beijing Institute of Technology ,E-mail: hiaaron@sina.cn

Received date: March 20,2017;Accepted date : April 15,2017; Published date: May 05,2017.

Citation for this Article: Zhang $\mathrm{H}$, Role of Biorepositories in Personalized Medicine: Isolation and Processing of Primary Tumor Cells for the initiation Tumor Cultures, J Cancer Research and Cellular Therapeutics, Doi: 10.31579/2640-1053/009.

Copyright : (c) 2017 Zhang H. This is an open-access article distributed under the terms of The Creative Commons Attribution License, which permits unrestricted use, distribution, and reproduction in any medium, provided the original author and source are credited.

\begin{abstract}
The introduction of three-dimensional (3D) tumor cultures has revolutionized anticancer drug research as these cultures allow for the study of drug resistance mechanisms that cannot be explored in traditional two dimensional (2D) monolayer cultures. Discoveries in the 3D tumor culture field suggest that individualized drug sensitivity testing of solid tumor specimens through the establishment and use of 3D tumor cell cultures following tissue collection will become a routine service offered by modern tissue repositories as they expand from their traditional research role to active participation in personalized medicine. Unfortunately, most information related to 3D tumor cultures comes from studies using established tumor cell lines rather than primary tumor cultures. However, accumulation of genetic aberrations in cancer cell lines occurs with increasing number of passages severely limiting their usefulness for personalized medicine. There is only very limited information available concerning technologies and standard operating procedures for the efficient and routine isolation and processing of primary tumor cells for the establishment of 3D tumor cultures from solid tumor specimens. The purpose of this work was to review experimental data from the literature that may provide relevant information concerning the isolation and processing of primary tumor cells for the establishment of 3D tumor cultures. Information reviewed here may help bio repositories in the development and standardization of technologies and standard operating procedures related to the use of 3D tumor cultures.
\end{abstract}

Keywords

Three-dimensional tumor culture; Personalized medicine; Primary tumor cell culture; Tissue repository, Drug sensitivity testing

\section{Introduction}

Accumulation of genetic aberrations in cancer cell lines occurs with increasing number of passages severely limiting their usefulness for personalized medicine [1,2]. In contrast, the critical importance of technologies related to the derivation, short term culture, and testing of primary tumor cells from solid tumors is increasingly recognized (for a recent review see 2). Short-term primary cultures of tumor cells derived from pieces of solid tumors have been used for predicting anticancer drug responses [3,4]. Observations suggest that individualized drug sensitivity testing of solid tumors through the isolation and culturing primary tumor cells in $3 \mathrm{D}$ may soon become routine and modern tissue repositories will need to be ready to support these activities as they expand from their traditional research role to active participation in personalized medicine [5].

Cancer cells grown in 3D cultures in a polymeric ECM closely mimic the biology of tumor development in vivo and numerous studies indicate that $3 \mathrm{D}$ cultures are superior to traditional 2D monolayer cultures for studies of key cellular behaviors like differentiation, proliferation, invasion and apoptosis [6-8]. Cancer cells grown in 3D culture are more resistant to chemotherapeutic agents, radiation and oncolytic virotherapy than cells in 2D culture and 3D tumor cell cultures are useful for preclinical evaluation of the cytotoxic effect of anticancer agents [6, 9-16].

Despite the significant advances in our understanding of various aspects of cancer initiation, progression, metastasis, tumor microenvironment and cancer stem cells we have achieved limited clinical success.
According to the current figures published by the American Cancer Society, more than half a million people die every year due to cancer in the USA alone.

\section{Methods and materials}

A review of the English language literature related to the isolation and processing of primary tumor cells for the establishment of 3D tumor cultures from solid tumor specimens was performed.

\section{Results}

Only a limited number of reports are available related to the isolation and processing of primary tumor cells for the establishment of $3 \mathrm{~d}$ tumor cultures from solid tumor specimens. However, these relate to colorectal, lung and brain tumors indicating that isolation and growth of primary tumors cells in $3 \mathrm{D}$ cultures is possible for a variety of solid tumors. The protocols have an initial step of mechanical mincing of tissues followed by at least some disruption of the original extracellular matrix by enzymatic dissociation. Isolated tumor cells or fragments can be grown and analyzed in a wide variety of $3 \mathrm{D}$ culture systems. Four prominent examples are reviewed below.

\section{Example 1. Kondo J et al. 2011 [17].}

Kondo et al found that retaining cell-cell contact enables preparation and culture of spheroids composed of pure primary cancer cells from colorectal cancer specimens that retain the characteristics of the parental tumors, grow in vitro in 3D cultures and could be used for personalized diagnostic applications, including chemosensitivity assays. 
To successfully maintain the cell-cell contact, they used Liberase DH (Roche Diagnostics) as a blend of digestion enzymes. They optimized the enzyme concentration and the digestion time to avoid overdigestion of colorectal tissues. Cancer specimens from colorectal cancer patients were mechanically and enzymatically digested and separated into two fractions using a cell strainer: the organoid fraction (ORG), which was retained in a 40-?m strainer, and the flow-through fraction (FT), which passed through the strainer. The FT fraction mainly contained single cells and many of the cells with epithelial markers were dead. In contrast, the ORG contained irregular sheet- or tube-like structures, which were termed organoids. After overnight culture, the organoids became spherical and bright with a smooth surface and were termed cancer tissue-originated spheroids (CTOSs). During formation, the CTOSs were draped with cellular debris that easily detached with pipetting. The diameter of the CTOSs was ?40$500 ? \mathrm{~m}$, depending on the cell strainer size. A CTOS with a diameter of $100 ? \mathrm{~m}$ consisted of ?100 cells. The authors were able to prepare CTOSs in most of the colorectal cancer specimens regardless of the disease stage and histology. CTOSs were highly viable with only a few dead cells on the outside. CTOSs could be cultured and expanded in vitro. Extracellular matrix was critical for favorable growth of CTOSs. Excellent proliferation was accomplished with the use of a 3D type I collagen-based culture system and serum-free medium designed for embryonic stem cells. The success rate for growth was $73.5 \%$. CTOSs were composed of highly purified and viable cancer cells, that retained the characteristics of the parental tumors, grew in vitro and could be used for personalized diagnostic applications, including chemosensitivity assays.

This study demonstrated the usefulness of cancer tissue-originated spheroid (CTOS) method for the primary culture of lung cancer cells. Surgical specimens and pleural effusion samples from lung cancer patients were minced with a scalpel into approximately one square $\mathrm{mm}$ pieces, and washed with Hank's balanced salt solution. Specimens were then digested in DMEM supplemented with Liberase $\mathrm{DH}$, at $37^{\circ} \mathrm{C}$ for 1 to 2 hours with gentle stirring by a magnetic bar. Digested tissue suspensions were passed through 500-?m and $250-? \mathrm{~m}$ metal mesh filters to remove large masses of undigested fragments. Suspensions were further filtered through 100-?m and 40-?m cell strainers. Fragments on the cell strainer and cells in the flow-through fractions were collected separately, and were each washed with HBSS and cultured in StemPro hESC medium in a nontreated dish. Pleural effusions were transferred to 50-ml tubes and centrifuged at $200 \mathrm{~g}$. Pellets were resuspended in HBSS, filtered through 40-?m cell strainers, and collected and cultured in the same manner as surgical specimens. CTOSs were embedded in Matrigel GFR and cultured in 100 ?l of basal medium containing growth factors (NRG1, LongIGF1, bFGF , Activin A, or EGF). Basal medium consisted of DMEM/F12, 2\% BSA fraction $\mathrm{V}$, nonessential amino acids, penicillin, streptomycin, ascorbic acid, human transferrin, ?-mercaptoethanol, and trace elements. Lung carcinoma CTOSs grew in vitro and could be used for personalized chemosensitivity assays.

\section{Chemical dissociation}

Numerous types of cations (such as $\mathrm{Ca}^{2+}$ and $\mathrm{Mg}^{2+}$ ) are used to maintain of cell surface integrity and the intracellular structural matrix [Li WC]. Chemical dissociation is a process where sequestration of these compounds from epithelial cells has been used to loosen intercellular bonds [Castell JV]. Sequestration is best achieved by exposure to EDTA or EGTA or complexes of tetraphenylboron plus potassium ions, which have been used to dissociate liver tissue, intestinal crypt cells and solid mammary tissue [ Harris CC]. Besides chelation, hypertonic solutions of disaccharides (sucrose, maltose, lactose, and cellobiose) have been reported to split gap junctions and zona occludentes, whose presence may be responsible for the clusters of cells that sometimes remain after enzymatic tissue digestion [Goodenough DA].

\section{Mechanical dissociation}

Mechanical dissociation of tissue involves repeated mincing with scissors or sharp blades, scraping the tissue surface, homogenization, filtration through a nylon or steel mesh (50 to $100 \mu \mathrm{m}$ opening), vortexing, repeated aspiration through pipettes or sequentially smaller needles (e.g., 16-,20-, and 23-gage), application of abnormal osmolarity stress, or any combination of these techniques [Cunningham RE]. Usually, tumor specimens are first minced into small pieces $(\sim 1 \mathrm{~mm})$ and then washed in tissue-specific medium to remove loosely bound cells or non-specific debris by gentle agitation. This process generates single cell suspension quickly with minimal number of steps. However, dissociation of tumor tissue using mechanical methods is not a suitable technique, for obtaining tumor cells for culture because the technique results in a high percentage of dead cells which secrete degrading enzymes

\section{Discussion}

Accumulation of genetic aberrations in cancer cell lines occurs with increasing number of passages severely limiting their usefulness for personalized medicine Research reviewed here indicates that isolation and culturing of primary tumor cells in $3 \mathrm{D}$ is possible and it is likely that primary 3D tumor cultures will soon be routinely used for individualized solid tumor testing. Modern tissue repositories will need to be ready to support these activities as they expand from their traditional research role to active participation in personalized medicine.

\section{References}

1. Gillet JP, Calcagno AM, Varma S, Marino M, Green LJ et al. (2011) Redefining the relevance of established cancer cell lines to the study of mechanisms of clinical anti-cancer drug resistance. PNAS USA.

2. Mitra A, Mishra L, Li S (2013) Technologies for deriving primary tumor cells for use in personalized cancer therapy. Trends Biotechnol 31: 347-354.

3. Brower SL, Fensterer JE, Bush JE (2008) The ChemoFx assay: an ex vivo chemosensitivity and resistance assay for predicting patient response to cancer chemotherapy. Methods Mol Biol 414: 57-78.

4. Jain KK (2009) Textbook of personalized medicine. Springer.

5. Valyi-Nagy K (2013) Three-dimensional tumor cell cultures and the role of tissue biorepositories in personalized medicine. J Clin Anat Pathol 1: 1-2.

6. Xu F, Burg KJ (2007) Three-dimensional polymeric systems for cancer cell studies. Cytotechnology 54: 135-143.

7. Page H, Flood P, Reynaud EG (2013) Three-dimensional cell cultures: current trends and beyond. Cell Tissue Res 352: 123-131.

8. Kimlin LC, Casagrande G, Virador VM (2013) In vitro threedimensional (3D) models in cancer research: an update. Mol Carcinog 52: 167-182.

9. Breslin S, O'Driscoll L (2013) Three-dimensional cell culture: the missing link to drug discovery. Drug Discov Today 18: 240-249.

10. Smalley KS , Lioni M, Herlyn M (2006) Life isn't flat: taking cancer biology to the next dimension. In Vitro Cell Dev Biol Anim 42: 242247.

11. Valyi-Nagy K, Folberg R, Valyi-Nagy T, Maniotis A (2007) Susceptibility of uveal melanoma to herpes simplex virus type 1: the role of tumor invasiveness, the extracellular matrix and chromatin sequestration. Exp Eye Res 84: 991-1000.

12. Valyi-Nagy K, Dosa S, Kovacs SK, Bacsa S, Voros A et al. (2010) Identification of virus resistant tumor cell subpopulations in three dimensional uveal melanoma cultures. Cancer Gene Ther 17: 223234.

13. Valyi-Nagy K, Voros A, Gagyi E, Valyi-Nagy T (2011) Increased Resistance of Vasculogenic Mimicry-Forming Uveal Melanoma Cells against Cytotoxic Agents in Three-Dimensional Cultures, Research on Melanoma - A Glimpse into Current Directions and Future Trends, Mandi Murph (Ed.), InTech, Chapter 18, 377-392.

14. Valyi-Nagy K, Kormos B, Ali M, Shukla D, Valyi-Nagy T (2012) Stem cell marker CD271 is expressed by vasculogenic mimicryforming uveal melanoma cells in three-dimensional cultures. Mol Vis 18: 588-592. 
15. Voros A, Kormos B, Valyi-Nagy T, Valyi-Nagy K (2013) Increased resistance of breast, prostate and embryonic carcinoma cells against herpes simplex virus in three-dimensional cultures. ISRN Oncol, Article ID 104913.

16. Xu Z, Gao Y, Hao Y, Li E, WangY et al. (2013) Application of a microfluidic chip-based 3D co-culture to test drug sensitivity for individualized treatment of lung cancer. Biomaterials 34 (2013) 4109-4117.

17. Kondo J, Endo H, Okuyama H, Osamu Ishikawa O, Iishi H et al. (2011) Retaining cell-cell contact enables preparation and culture of spheroids composed of pure primary cancer cells from colorectal cancer. Proc Natl Acad Sci U S A 108: 6235-6240.

18. Endo H, Okami J, Okuyama H, Kumagai $T$, Uchida $J$ et al. (2013) Spheroid culture of primary lung cancer cells with neuregulin 1/HER3 pathway activation. J Thorac Oncol. 8: 131139.

19.
Jiquet Jiglaire C, Baeza-Kallee N, Denicolaï E, Barets D, Metellus P et al. (2014) Ex vivo cultures of glioblastoma in three-dimensional hydrogel maintain the original tumor growth behavior and are suitable for preclinical drug and radiation sensitivity screening. Exp Cell Res 321: 99-108.

20. Castell JV, Gomez-Lechon MJ. Liver cell culture techniques. Methods Mol Biol. 2009;481:35-46.

21. Li WC, Ralphs KL, Tosh D. Isolation and culture of adult mouse hepatocytes. Methods Mol Biol. 2010;633:185-96.

22. Goodenough DA, Gilula NB. The splitting of hepatocyte gap junctions and zonulae occludentes with hypertonic disaccharides. J Cell Biol. 1974;61(3):575-90.

23. Cunningham RE. Tissue disaggregation. Methods Mol Biol. 2010;588:327-30. 